\title{
Corpus
}

\section{Yana GRINSHPUN. - Ô entre langue(s), discours et graphie. Paris / Gap : Ophrys (« Langues, langage et textes »), 2008, 314 pages.}

\section{Michèle Monte}

\section{(2) OpenEdition}

Édition électronique

URL : http://journals.openedition.org/corpus/1777

DOI : $10.4000 /$ corpus. 1777

ISSN : 1765-3126

Éditeur

Bases; corpus et langage - UMR 6039

\section{Édition imprimée}

Date de publication : 15 novembre 2009

ISSN : 1638-9808

\section{Référence électronique}

Michèle Monte, "Yana grinshpun. - Ô entre langue(s), discours et graphie. Paris / Gap : Ophrys

("Langues, langage et textes »), 2008, 314 pages. », Corpus [En ligne], 8| 2009, mis en ligne le 01 juillet 2010, consulté le 21 septembre 2020. URL : http://journals.openedition.org/corpus/1777 ; DOI :

https://doi.org/10.4000/corpus. 1777

Ce document a été généré automatiquement le 21 septembre 2020.

(c) Tous droits réservés 


\title{
Yana GRINSHPUN. - Ô entre langue(s), discours et graphie. Paris / Gap : Ophrys (« Langues, langage et textes »), 2008, 314 pages.
}

\author{
Michèle Monte
}

Le livre de Yana Grinshpun est consacré à une particule qui a peu attiré l'attention jusqu'ici, probablement parce que son usage est réservé au discours écrit littéraire. Dans une première partie historique, l'auteure présente sommairement l'emploi de la particule en grec ancien, en latin et en français puis analyse son traitement dans les grammaires depuis l'Antiquité et dans les dictionnaires français du XVI ${ }^{e}-X_{X}{ }^{e}$ siècles. Outre la caractérisation incertaine de $\hat{o}$ - tantôt considéré comme interjection, tantôt comme particule, ou adverbe, voire indice du vocatif -, ce parcours relève deux difficultés qui ne sont pas sans lien: les hésitations entre les deux graphies oh et $\hat{o}$ et l'utilisation de ô dans deux contextes différents, devant des syntagmes en apostrophe d'une part, dans des énoncés exclamatifs d'autre part. On observe progressivement une clarification : oh est utilisé préférentiellement pour l'emploi interjectif indépendant ou devant un énoncé exclamatif introduit par un morphème en qu-, $\hat{o}$ apparaît pour l'essentiel devant apostrophe et devant GN exclamatif sans qu-. Cependant cet effort des grammairiens et typographes pour répartir strictement les emplois se heurte à l'ambiguïté de $\hat{o}$, qui ne rend pas la tâche facile aux scripteurs, et à l'impact des genres sur la graphie, ô empiétant sur oh en poésie, et oh sur ô dans les comédies et la correspondance. Il existe une tension entre la répartition selon les genres ou registres et la répartition selon des critères syntaxiques.

Une fois menée cette enquête historique passionnante qui montre comment les usagers et les lettrés résolvent progressivement un problème d'homonymie, Yana Grinshpun aborde dans la partie centrale, qui représente la moitié de l'ouvrage, la difficile question du fonctionnement sémantique et syntaxique de ô. Après un rapide parcours des définitions de l'interjection chez différents grammairiens et linguistes, elle en 
conclut que ô n'ayant pas de sens sans le GN qu'il introduit ne peut être considéré comme une interjection. L'analyse d'un vaste corpus lui permet d'établir que $\hat{o}$ introduit soit des pronoms (P2 et P5 surtout) suivis ou non d'expansions relatives ou d'appositions, soit des GN à déterminant zéro ou introduits par un possessif. L'emploi avec l'article défini, beaucoup plus réduit, se trouve essentiellement dans des énoncés exclamatifs, l'emploi avec le démonstratif est rare et ne s'observe qu'à partir du XIX ${ }^{\mathrm{e}}$ siècle. Yana Grinshpun propose de distinguer syntaxiquement deux cas de figure : celui où le segment $\hat{o}+\mathrm{GN}$ est dépendant d'un élément co(n)textuel et celui où il est autonome. Chacun de ces deux cas offre 2 possibilités sémantiques: en emploi dépendant, une valeur invocative (ô prince, j'implore ta pitié.) et une valeur prédicative (Il est parti. ô sage décision!) et en emploi autonome, une valeur interjective (Il est venu, $\hat{o}$ ciel, pour attaquer la ville.) et une valeur évocative (ô Troyens ! ô mon père!).

L'emploi invocatif offre des spécificités intéressantes par rapport à l'apostrophe ordinaire : $\hat{o}+$ GN doit s'appuyer sur une sélection préalable de l'allocutaire et sert à prédiquer une propriété de celui-ci plus qu'à l'identifier. Le signifié du $\mathrm{N}$ doit renvoyer à une catégorisation bien stabilisée ou être légitimé par l'acte d'énonciation. Ceci se rapproche du rôle commentatif et évaluatif de $\hat{o}+\mathrm{GN}$ en emploi prédicatif par rapport à un support textuel ou situationnel (ô rage, ô désespoir, ô vieillesse ennemie!). L'emploi interjectif implique une prise à témoin qui concerne essentiellement la divinité, sans que celle-ci soit constituée en allocutaire (pas de coréférence avec une P2 dans l'énoncé-hôte). L'emploi évocatif correspond au surgissement d'une représentation dans l'esprit du locuteur, sans support ni apostrophe. Grinshpun fait remarquer qu'on peut regrouper les emplois invocatifs et interjectifs, d'une part (énoncés centrés sur l'allocutaire), les emplois prédicatifs et évocatifs, d'autre part (énoncés centrés sur le locuteur). Toutefois cette séparation n'est pas étanche, ce qui avait déjà été observé par Détrie $^{1}$ (2006) et Monte $^{2}$ (2005) et laisse intacte la question du signifié propre de $\hat{o}$.

Pour la résoudre, Grinshpun fait l'hypothèse que ô cumule une valeur interpellative et la valeur exclamative de oh. Pour cerner celle-ci, elle propose une différenciation des interjections ah et oh en fonction de leurs contextes. Oh serait du côté d'un centrage sur le locuteur sans prise en compte préalable d'un autre point de vue. De là elle propose de voir dans ô une re-présentation d'un référent dont la catégorisation a déjà été validée, re-présentation opérant un renvoi au centre de la notion exprimée par le $\mathrm{GN}$, associée à la mise en spectacle d'une émotion et à une difficulté d'accès au destinataire. La scène énonciative construite par ô serait celle d'une interlocution sans véritable altérité où l'énoncé serait soustrait à toute confrontation de points de vue et présenté comme déjà validé par les deux coénonciateurs. Ô activerait le prototype associé au GN qui le suit, d'où son affinité avec les textes poétiques où le processus évocatif, si l'on adhère à la théorie de Dominicy, fait appel à des prototypes engrangés dans la mémoire. On ne voit pas trop en revanche ce qui, dans cette valeur sémantique, justifierait son emploi régulier dans les genres oratoires.

5 Une sous-partie consacrée aux caractéristiques phonétiques de ô ouvre des perspectives de travail intéressantes mais les résultats s'avèrent trop limités et contradictoires pour étayer véritablement les propositions antérieures.

Quant aux deux dernières sous-parties, consacrées aux emplois discursifs de $\hat{o}$ et à l'étude de traductions en français de l'allemand et de l'anglais où des occurrences de $\hat{o}$ sont rajoutées par rapport au texte original, elles mettent en évidence d'une part que $\hat{o}$ est en partie idiolectal depuis le XIX ${ }^{\mathrm{e}}$ siècle, d'autre part qu'il est souvent choisi par les 
traducteurs lorsqu'il s'agit d'invoquer des absents ou des êtres inanimés, ce que Grinshpun met en rapport avec la difficulté d'accès au destinataire qui serait un des traits sémantiques de cette particule.

7 La dernière partie, qui représente un quart de l'ouvrage, est consacré à la situation de ô au XVII ${ }^{e}$ siècle. Après une présentation du corpus (tragédies, comédies, sermons et oraisons funèbres, plaidoyers, poésies, romans, méditations religieuses), l'auteure présente les résultats statistiques concernant les contraintes distributionnelles et les emplois de ô. Les emplois invocatifs prédominent largement sauf dans la tragédie où la fréquence de ô ciel, ô $\operatorname{dieu}(x)$ fait passer en premier les emplois interjectifs. Grinshpun analyse ensuite les emplois au théâtre en insistant sur les paramètres génériques (tragédie vs comédie, vers vs prose) et sur le fait que, dans les emplois invocatifs, l'allocutaire est pris à témoin mais n'est pas traité en interlocuteur. Enfin l'étude des emplois de ô par Corneille et Racine dans leurs tragédies, Massillon et Bossuet dans leurs sermons, fait apparaître, par-delà les différences génériques attendues, des spécificités idiolectales que l'auteure relie très finement à des positionnements esthétiques et religieux différents.

Grinshpun insiste dans la conclusion sur le fait que ô possède une valeur unique et que l'interprétation résulte de l'interaction de cette valeur avec le contexte. Cette valeur abstraite de re-présentation catégorisante d'une entité déjà construite implique, selon elle, une certaine scène d'énonciation, ce qui explique la spécialisation de ô dans certains genres.

9 Ayant moi-même travaillé sur l'emploi de ô et de l'apostrophe (cf. note 2), j'ai trouvé un grand intérêt dans ce livre qui apporte beaucoup d'éléments précieux pour la connaissance de cette particule et dont la démarche (parcours historique, travail sur un vaste corpus, prise en compte des genres discursifs, étude d'exemples contextualisés, recherche d'un signifié unique, confrontation avec oh) emporte l'adhésion. Néanmoins, j'émettrai quelques réserves sur les résultats présentés. Tout d'abord, d'un point de vue formel, des coquilles plutôt fâcheuses dans les résultats statistiques nécessiteraient une feuille d'errata pour faciliter la lecture de certains tableaux. Le plan choisi n'évite pas de nombreuses répétitions un peu fastidieuses ainsi que quelques contradictions d'une partie à l'autre. Ensuite, le concept d'autonomie de $\hat{o}+\mathrm{GN}$ oscille entre une perspective syntaxique et une perspective textuelle, ce qui conduit à des classements peu cohérents. Par ailleurs, l'attention portée aux genres discursifs conduit souvent Yana Grinshpun à minorer les exigences du contexte étroit, de l'interaction spécifique de tel ou tel endroit du texte. Les analyses discursives proposées sont souvent trop systématiques. Ainsi la difficulté d'accès au destinataire me semble un trait discutable du signifié de $\hat{o}$, dont le lien avec la valeur abstraite de la particule n'est jamais clairement argumenté, et dont les exemples fournis ne sont à mon sens pas très probants. Si la notion culiolienne de renvoi au centre de la notion me paraît plus convaincante, il faudrait sans doute l'étayer davantage et surtout l'articuler plus rigoureusement à l'observation, confirmée par mes propres analyses, que la scène d'énonciation construite par ô est consensuelle et ne différencie pas les deux pôles de l'interaction, de sorte que ô aurait précisément pour effet de gommer dans certains cas la difficulté d'accès au destinataire en supposant acquise sa présence. Mais ô se contente-t-il de réactiver l'accord du coénonciateur en présentant comme déjà validés soit la catégorisation qui lui est appliquée dans le GN invocatif, soit l'évaluation qui est 
faite dans l'énoncé pour lequel il est pris à témoin, soit l'acte de langage effectué, ou sollicite-t-il à nouveau son accord ? Ce point devrait être approfondi.

En dépit de ces réserves, on ne peut que saluer la démarche de Yana Grinshpun et souhaiter que ses travaux soient diffusés, discutés et poursuivis. Avec ô la langue écrite s'est en effet dotée d'un outil complexe, bien différent sur le plan syntaxique de particules telles que bon ou eh bien, mais tout aussi riche sur le plan du positionnement énonciatif.

\section{NOTES}

1. De la non-personne à la personne : l'apostrophe nominale, CNRS éditions (cf. compte-rendu dans Corpus $\left.n^{\circ} 7\right)$.

2. " $\hat{O}+\mathrm{SN}$ dans les textes poétiques : entre apostrophe et exclamation », Modèles linguistiques, tome XXVI-2, vol. $52: 45-68$.

\section{AUTEUR}

\section{MICHĖLE MONTE}

Université du Sud Toulon-Var 